\title{
Modulation Instability of Ion-Acoustic Waves in Plasma with Nonthermal Electrons
}

\author{
Basudev Ghosh ${ }^{1}$ and Sreyasi Banerjee ${ }^{2}$ \\ ${ }^{1}$ Department of Physics, Jadavpur University, Kolkata 700 032, India \\ ${ }^{2}$ Department of Electronics, Vidyasagar College, Kolkata 700 006, India \\ Correspondence should be addressed to Basudev Ghosh; bsdvghosh@gmail.com
}

Received 2 May 2014; Accepted 3 July 2014; Published 16 July 2014

Academic Editor: Milan S. Dimitrijevic

Copyright ( 2014 B. Ghosh and S. Banerjee. This is an open access article distributed under the Creative Commons Attribution License, which permits unrestricted use, distribution, and reproduction in any medium, provided the original work is properly cited.

\begin{abstract}
Modulational instability of ion-acoustic waves has been theoretically investigated in an unmagnetized collisionless plasma with nonthermal electrons, Boltzmann positrons, and warm positive ions. To describe the nonlinear evolution of the wave amplitude a nonlinear Schrödinger (NLS) equation has been derived by using multiple scale perturbation technique. The nonthermal parameter, positron concentration, and ion temperature are shown to play significant role in the modulational instability of ion-acoustic waves and the formation of envelope solitons.
\end{abstract}

\section{Introduction}

Electron-positron-ion $(e-p-i)$ plasmas occur in many astrophysical environments such as active galactic nuclei [1], pulsar magnetospheres [2], polar regions of neutron stars [3], centres of our galaxy [4], the early universe [5, 6], and the solar atmosphere [7]. For this, over the last two decades there has been a great deal of interest in the study of nonlinear wave phenomena in $e-p-i$ plasmas [8-12]. Positrons are produced by pair production in high energy processes occurring in many astrophysical environments. Popel et al. [9] have reported decrease in soliton amplitude in the presence of positrons. Jehan et al. [13] have shown that solitons become narrower as the concentration of positron increases. The presence of non-Maxwellian electron is common in space and astrophysical plasmas including the magnetosphere [12] and auroral zones [14]. The presence of such non-Maxwellian electrons gives rise to many interesting characteristics in the nonlinear propagation of waves including the ion-acoustic solitons $[15,16]$. The solitary structures with density depression in the magnetosphere observed by the Freja satellites $[17,18]$ have been explained by Cairns et al. [19] by assuming electron distribution to be nonthermal. Nonlinear ion-acoustic solitary waves in $e-p-i$ plasma have been considered by some authors $[9,20,21]$ assuming ions to be cold. In practice ions have finite temperature and the ionic temperature can significantly affect the characteristics of nonlinear ion-acoustic structures [10, 22, 23]. Chawla et al. [24] have considered ion-acoustic waves in $e$ - $p$ - $i$ plasma with warm adiabatic ions and isothermal electrons. Baluku and Hellberg [25] have considered ion-acoustic solitary waves in $e-p-i$ plasma with cold ions and nonthermal electrons. Hence it is interesting to study the nonlinear ion-acoustic waves in $e-p-i$ plasma assuming simultaneous presence of nonthermal electrons, warm negative ions, and the positrons. Recently Pakzad [11] has shown that the presence of warm ions and nonthermal electrons can modify parametric regions of existence of ion-acoustic solitary waves. A nonlinear theory of ion-acoustic waves in $e-p$ - $i$ plasma has been developed by Dubinov and Sazonkin [26] considering polytropic laws of compression and rarefraction for all plasma components. Survey of the past literatures shows that a large number of works on KdV type and large amplitude solitary structure formation in $e-p-i$ plasmas have been reported. Nonlinear propagation of waves in a dispersive medium is generically subject to amplitude modulation due to carrier wave selfinteraction or intrinsic nonlinearity of the medium. Modulational instability is an important phenomenon in connection 
with stable wave propagation. However, only a few works have been reported in recent years on the modulational instability and formation of envelope soliton in $e-p$ - $i$ plasmas $[20,21$, 24]. It has been shown that the presence of positrons shifts the critical wave number separating the stability and instability regions to higher values and for fixed amplitude, width of envelope solitons decreases with the increase of positron concentration. Mahmood et al. [27] have studied modulational instability of ion-acoustic waves in $e-p-i$ plasma with warm ions and isothermal electrons and positrons at the same temperature. Chawla et al. [24] have studied the effects of ion temperature, positron concentration, and positron temperature on the modulational instability of ionacoustic waves in $e-p-i$ plasma with isothermal electrons and positrons at different temperatures. Bains et al. [28] have considered modulational instability of ion-acoustic waves in $e-p-i$ plasma with dust particles. Eslami et al. [29] have considered modulational instability of ion-acoustic waves in $e-p-i$ plasma with electrons and positrons following qnonextensive distribution. Gill et al. [21] have studied modulational instability of ion-acoustic waves in $e-p-i$ plasma with superthermal electrons and isothermal positrons. Zhang et al. [30] have investigated modulational instability of ionacoustic waves in $e-p$ - $i$ plasma with nonthermally distributed electrons and cold ions. Modulational instability and excitation of ion-acoustic envelope solitons in $e-p-i$ plasma with nonthermal electrons have been investigated by Gill et al. [31] including ion temperature. The purpose of the present paper is to make a detailed study of modulational instability of ionacoustic waves in $e$ - $p$ - $i$ plasma including simultaneously both the effects of nonthermality of electrons and ion-temperature.

\section{Basic Formulation}

We consider an unmagnetized collisionless plasma consisting of warm positive ions, Boltzmann positrons, and nonthermal electrons. The normalized basic equations governing ion dynamics for one-dimensional propagation in such plasma in dimensionless form are as follows [28]:

$$
\begin{gathered}
\frac{\partial n_{i}}{\partial t}+\frac{\partial}{\partial x}\left(n_{i} v_{i}\right)=0 \\
\frac{\partial v_{i}}{\partial t}+v_{i} \frac{\partial v_{i}}{\partial x}+\frac{3 \sigma_{i}}{(1-\chi)^{2}} n_{i} \frac{\partial n_{i}}{\partial x}=-\frac{\partial \phi}{\partial x} \\
\frac{\partial^{2} \phi}{\partial x^{2}}=n_{e}-n_{p}-n_{i} .
\end{gathered}
$$

In aforementioned equations, the parameters $n_{i}, v_{i}$ are, respectively, the concentration and velocity of the positive ions; $n_{e}$ and $n_{p}$ are, respectively, the concentration of electrons and positrons; $\phi$ denotes the electrostatic potential; other parameters have their usual meaning. Different quantities are normalized as follows: the velocities by ion-acoustic speed $C_{s}=\sqrt{k_{B} T_{e} / m_{i}}$, the densities by equilibrium electron density $n_{e 0}$, all the length $x$ by the electron Debye length $\lambda_{\mathrm{De}}=\sqrt{k_{B} T_{e} / 4 e^{2} n_{e 0}}$, time by $\lambda_{\mathrm{De}} / C_{s}$, ion temperature $T_{i}$ by $T_{e}\left(\sigma_{i}=T_{i} / T_{e}\right)$ and the potential $\phi$ by $k_{B} T_{e} / e$, where $k_{B}$ is the Boltzmann's constant. The nonthermal electron density is given by [19]

$$
n_{e}=\left(1-\beta \phi+\beta \phi^{2}\right) \exp (\phi)
$$

where $\beta=4 \delta /(1+3 \delta)$ measures the deviation from the thermalized state and $\delta$ determines the presence of nonthermal electrons inside the plasma. The density of Boltzmann positrons is given by

$$
n_{p}=\chi \exp \left(-\sigma_{p} \phi\right)
$$

where $\chi=n_{p 0} / n_{e 0}$ is the ratio between the unperturbed positron and electron number densities and $\sigma_{p}=T_{e} / T_{p}$ is the ratio between electron and positron temperatures. The equilibrium charge neutrality condition in normalized form is given by

$$
\chi+n_{i 0}=1
$$

in which $n_{i 0}$ is the equilibrium ion density normalized by the equilibrium electron density.

Using (2) and (3), Poisson's equation in (1) is rewritten as

$$
\frac{\partial^{2} \phi}{\partial x^{2}}=\left(1-\beta+\beta \phi^{2}\right) \exp (\phi)-\chi \exp \left(-\sigma_{p} \phi\right)-n_{i} \text {. }
$$

\section{Derivation of the Evolution Equation}

Following the usual procedure we make the following Fourier expansions for the field quantities [28, 32-34]:

$$
F=\varepsilon^{2} F_{0}^{\prime}+\sum_{s=1}^{\infty} \varepsilon_{s}\left\{F_{s} \exp (i s \psi)+F_{s}^{*} \exp (-i s \psi)\right\}
$$

where $F$ stands for the field quantities $n_{i}, v_{i}$, and $\phi ; F_{0}^{\prime}$ and $F_{s}$ are assumed to vary slowly with space and time; that is, they are supposed to be functions of $\xi=\varepsilon\left(x-C_{g} t\right)$ and $\tau=\varepsilon^{2} \tau$, with $\varepsilon$ being a small parameter and $C_{g}$ the group velocity; $\psi=k x-\omega t$ ( $\omega, k$ being two constants satisfying linear dispersion relation). Substituting the expansion (6) in (1) and (5) and then equating from both sides the coefficients of $\exp (i \psi), \exp (2 i \psi)$, and terms independent of $\psi$ we obtain three sets of equations which we call, respectively, I, II, and III. To solve these equations we make the following perturbation expansion for the field quantities, $F_{0}^{\prime}$ and $F_{s}$, which we denote by $X$ :

$$
X=X^{(1)}+\varepsilon X^{(2)}+\varepsilon X^{(3)}+\cdots
$$

Solving the lowest order equations obtained from the set of equations I after substituting the expansion (7) we get the following solutions for the first harmonic quantities in the lowest order:

$$
\begin{aligned}
& n_{i 1}^{(1)}=\left(1-\beta+\chi \sigma_{p}+k^{2}\right) \cdot \alpha, \\
& v_{i 1}^{(1)}=\frac{\omega \cdot\left(1-\beta+\chi \sigma_{p}+k^{2}\right)}{(1-\chi)} \cdot \alpha,
\end{aligned}
$$


where

$$
\alpha=\phi_{1}^{(1)}
$$

The linear dispersion relation is obtained as

$$
\omega^{2}=k^{2}\left[\frac{(1-\chi)}{\left(1-\beta+\chi \sigma_{p}+k^{2}\right)}+\frac{3 \sigma_{i}}{(1-\chi)}\right] \text {. }
$$

The wave frequency is found to increase with the increase in the nonthermal parameter $\beta$ and the ion temperature. On the other hand, increase in positron concentration decreases the wave frequency. In this connection it is pertinent to mention that Pakzad [35] reported an incorrect result and it was pointed out and corrected by Baluku and Hellberg [25]. If we put $\beta=0, \chi=0$, and $\sigma_{i}=0$, we get the linear dispersion relation for ion-acoustic waves in $e-i$ plasma as obtained by Kakutani and Sugimoto [36]. In the limit $k \rightarrow 0$ (10) leads to the normalized ion-acoustic speed $\left(V_{s}\right)$ modified by the presence of positrons, ion-temperature, and non-Maxwellian electron distribution:

$$
V_{s}^{2}=\frac{(1-\chi)}{\left(1-\beta+\chi \sigma_{p}\right)}+\frac{3 \sigma_{i}}{(1-\chi)} .
$$

It agrees with the results obtained by Baluku and Hellberg [25] for the case of cold ions $\left(\sigma_{i}=0\right)$. Equation (11) shows that, for the case of cold ions, increase in positron concentration decreases the phase speed [15], increase in the nonthermal parameter $(\beta)$ leads to an increase in phase speed, and also increase in ion temperature increases the phase speed.

First harmonic quantities in the second order are obtained from the solutions (8) by replacing $-i \omega$ by $-i \omega-$ $\varepsilon C_{g}(\partial / \partial \xi)+\varepsilon^{2}(\partial / \partial \tau)$ and $i k$ by $i k+\varepsilon(\partial / \partial \xi)$ and then picking out order $\varepsilon$ terms. These are as follows:

$$
\begin{gathered}
\phi_{1}^{(2)}=0 \\
n_{i_{1}}^{(2)}=-i 2 k \frac{\partial \alpha}{\partial \xi}, \\
v_{i_{1}}^{(2)}=\left[\left\{\left(\frac{\omega}{k^{2}}-\frac{C_{g}}{k}\right)\left(\frac{1-\beta+\chi \sigma_{p}}{1-\chi}\right)\right\}\right. \\
\left.-\frac{2 \omega k}{1-\chi}-\frac{k^{2} C_{g}}{1-\chi}\right] \frac{\partial \alpha}{\partial \xi} .
\end{gathered}
$$

The second harmonic quantities in the lowest order obtained from the set of equations II after substituting the expansion (7) are as follows:

$$
\begin{aligned}
& \phi_{2}^{(1)}=A_{1} \cdot \alpha^{2}, \\
& n_{i_{2}}^{(1)}=\left[\left\{A_{1}\left(1-\beta+\chi \sigma_{p}+4 k^{2}\right)^{2}\right\}+\left\{\frac{\chi \sigma_{p}^{2}}{2}-\beta\right\}\right] \cdot \alpha^{2},
\end{aligned}
$$

$$
\begin{aligned}
v_{i_{2}}^{(1)}=\frac{\omega}{k(1-\chi)}[ & \left\{A_{1}\left(1-\beta+\chi \sigma_{p}+4 k^{2}\right)\right\} \\
& -\frac{\left(1-\beta+\chi \sigma_{p}+k^{2}\right)^{2}}{(1-\chi)} \\
& \left.+\left(\frac{\chi \sigma_{p}^{2}}{2}-\beta\right)\right] \cdot \alpha^{2},
\end{aligned}
$$

where

$$
\begin{aligned}
A_{1}= & {\left[\left(\frac{2 \omega^{2}}{k(1-\chi)}-\frac{6 \sigma_{i}}{(1-\chi)}\right)\left(\frac{\chi \sigma_{p}^{2}}{2}-\beta\right)\right] } \\
- & {\left[\left(\frac{3 \omega^{2}}{k(1-\chi)^{2}}-\frac{3 \sigma_{i}}{(1-\chi)^{2}}\right)\left(1-\beta+\chi \sigma_{p}+k^{2}\right)^{2}\right] } \\
& \times\left(\left[\frac{6 \sigma_{i} k}{(1-\chi)}\left(1-\beta+\chi \sigma_{p}+4 k^{2}\right)\right]+2 k\right. \\
& \left.-\frac{\left[2 \omega^{2}\left(1-\beta+\chi \sigma_{p}+4 k^{2}\right)\right]}{k(1-\chi)}\right)^{-1} .
\end{aligned}
$$

The zeroth harmonic components generated through nonlinear self-interaction of the finite amplitude wave are obtained from the set of equations III after substituting the expansion (7):

$$
\begin{aligned}
\phi_{0}^{(1)}= & B_{1} \cdot \alpha \alpha^{*}, \\
n_{i_{0}}^{(1)}= & {\left[\left\{B_{1}\left(1+\chi \sigma_{p}\right)\right\}-2 \beta\right] \cdot \alpha \alpha^{*}, } \\
v_{i_{0}}^{(1)}= & {\left[\left\{B_{1} \frac{C_{g}\left(1+\chi \sigma_{p}\right)}{1-\chi}\right\}-\frac{2 \beta C_{g}}{1-\chi}\right.} \\
& \left.-\frac{\left\{2 \omega\left(1-\beta+\chi \sigma_{p}+k^{2}\right)^{2}\right\}}{k(1-\chi)^{2}}\right] \alpha \alpha^{*},
\end{aligned}
$$

where

$$
\begin{aligned}
B_{1}= & \frac{6 \beta \sigma_{i}}{1-\chi}-\frac{2 \beta C_{g}^{2}}{1-\chi} \\
& -\left[\frac{\left(1-\beta+\chi \sigma_{p}+k^{2}\right)^{2}}{(1-\chi)^{2}}\left(3 \sigma_{i}+2 C_{g} \frac{\omega}{k}+\frac{\omega^{2}}{k^{2}}\right)\right] \\
& \times\left(1-\frac{C_{g}^{2}\left(1+\chi \sigma_{p}\right)}{1-\chi}+\left[\frac{3 \sigma_{i}}{1-\chi}\left(1+\chi \sigma_{p}\right)\right]\right)^{-1} .
\end{aligned}
$$


Now in order to derive the NLS equation, we need to consider first harmonic quantities in the third order. Collecting coefficients of $\varepsilon^{3}$ from both sides of the set of equations I after substituting perturbation expansion (7), we get a set of equations for first harmonic quantities in the third order from which after proper elimination we obtain the following desired NLS equation:

$$
\begin{gathered}
i \frac{\partial \alpha}{\partial \tau}+P \cdot \frac{\partial^{2} \alpha}{\partial \xi^{2}}=Q \cdot \alpha \alpha^{*}, \\
P=\frac{k(1-\chi)}{2 \omega\left(1-\beta+\chi \sigma_{p}+k^{2}\right)} \\
\times\left[\frac{2 \omega C_{g}}{1-\chi}-\frac{\omega^{2}}{k(1-\chi)}-\frac{2 \omega^{2}}{(1-\chi)^{2}}-\frac{k C_{g} \omega}{(1-\chi)^{2}}-C_{g}\right. \\
\quad \times\left[\left\{\left(\frac{\omega}{k^{2}}-\frac{C_{g}}{k}\right)\left(\frac{1-\beta+\chi \sigma_{p}}{1-\chi}\right)\right\}-\frac{2 \omega k}{1-\chi}-\frac{k^{2} C_{g}}{1-\chi}\right] \\
\\
\left.\quad-\frac{3 \sigma_{i} k}{(1-\chi)^{2}}+\frac{\omega}{k}\left\{\left(\frac{\omega}{k^{2}}-\frac{C_{g}}{k}\right)\left(\frac{1-\beta+\chi \sigma_{p}}{1-\chi}\right)\right\}\right]
\end{gathered}
$$$$
Q=\frac{k(1-\chi)}{2 \omega\left(1-\beta+\chi \sigma_{p}+k^{2}\right)}\left[F_{2} k-\frac{\omega^{2} F_{3}}{k(1-\chi)}+\frac{\omega F_{1}}{(1-\chi)}\right] \text {, }
$$

where

$$
\begin{aligned}
F_{1}= & {\left[\left(1+\chi \sigma_{p}\right) B_{1}-2 \beta\right] \frac{\omega\left(1-\beta+\chi \sigma_{p}+k^{2}\right)}{k(1-\chi)} } \\
& +\left(1-\beta+\chi \sigma_{p}+k^{2}\right) \\
& \times\left[\frac{C_{g}\left(1+\chi \sigma_{p}\right)}{1-\chi}-\frac{2 \beta C_{g}}{1-\chi}-\frac{2 \omega\left(1-\beta+\chi \sigma_{p}+k^{2}\right)^{2}}{k(1-\chi)^{2}}\right] \\
& \left.+\frac{\omega\left(1-\beta+\chi \sigma_{p}+k^{2}\right)}{k(1-\chi)}\right] \\
& \times\left[\frac{C_{g}\left(1+\chi \sigma_{p}\right)}{1-\chi}-\frac{2 \beta C_{g}}{1-\chi}-\frac{\left(1-\beta+\chi \sigma_{p}+k^{2}\right)^{2}}{1-\chi}\right] \\
& +\left[\frac{\omega\left(1-\beta+\chi \sigma_{p}+k^{2}\right)}{k(1-\chi)}\right] \\
& {\left.\left[1-\beta+\chi \sigma_{p}+4 k^{2}\right) A_{1}+\left(\frac{\chi \sigma_{p}^{2}}{2}-\beta\right)\right] }
\end{aligned}
$$

$$
\begin{aligned}
& F_{2}= {\left[\frac{C_{g}\left(1+\chi \sigma_{p}\right)}{1-\chi} B_{1}-\frac{2 \beta C_{g}}{1-\chi}-\frac{2 \omega\left(1-\beta+\chi \sigma_{p}+k^{2}\right)^{2}}{k(1-\chi)^{2}}\right] } \\
& \times \frac{\omega\left(1-\beta+\chi \sigma_{p}+k^{2}\right)}{k(1-\chi)} \frac{\omega^{2}\left(1-\beta+\chi \sigma_{p}+k^{2}\right)}{k^{2}(1-\chi)^{2}} \\
& \times\left[\left(1-\beta+\chi \sigma_{p}+4 k^{2}\right)+\left(\frac{\chi \sigma_{p}^{2}}{2}-\beta\right)\right. \\
&\left.+\frac{\left(1-\beta+\chi \sigma_{p}+k^{2}\right)^{2}}{(1-\chi)}\right] \\
& F_{3}= B_{1}\left(2 \beta+\chi \sigma^{2}\left(1-\beta+\chi \sigma_{p}^{2}+k^{2}\right)+\beta+\beta A_{1} .\right. \\
& \times\left[\left(1+\chi \sigma_{p}\right) B_{1}-2 \beta+\left(1-\beta+\chi \sigma_{p}+4 k^{2}\right) A_{1}\right. \\
&\left.+\left(\frac{\chi \sigma_{p}^{2}}{2}-\beta\right)\right]
\end{aligned}
$$

\section{Modulational Instability and Envelope Solitons}

NLS equation (17) describes the nonlinear evolution of the amplitude of IAWs in $e-p-i$ plasma with warm ions, nonthermal electrons, and Boltzmann positrons. NLS equation (17) has been studied extensively in connection with the nonlinear propagation of different wave modes. It is well known that a uniform wave train may be modulationally stable or unstable depending on the sign of the product of the group dispersive and the nonlinearity coefficient, that is, $P Q$. As the coefficients depend on the plasma parameters such as nonthermal parameter $\beta$, ion temperature $\sigma_{i}$, and positron concentration $\chi$, the product of $P Q$ can have both positive and negative values over different parametric regions. The wave is modulationally unstable if $P Q<0$ and the growth rate of instability has a maximum value $g_{m}$ given by

$$
g_{m}=|Q| \alpha_{0}^{2}
$$

where $\alpha_{0}$ is the constant real amplitude of the carrier wave. For $P Q>0$, the IAW is modulationally stable. As the product can have both positive and negative signs for different values of $\beta, \sigma_{i}$, and $\chi$, there are accordingly two types of localized solitary wave solutions of the NLS equation (17). To obtain the soliton profile we let

$$
\alpha=\rho \exp (i \theta),
$$


where $\rho$ and $\theta$ are two real variables. Solving the resulting equations for $\rho$ and $\theta$ with $P Q<0$ we get the following bright envelope soliton solution:

$$
\rho=\frac{\sqrt{2|P / Q|}}{L} \operatorname{sech}\left(\frac{\xi-U \tau}{L}\right),
$$

where $U$ is the envelope speed and $L$ is the spatial width of the pulse. It encloses high frequency carrier oscillations and vanishes at infinity. On the other hand, if $P Q>0$, a stable gray or dark soliton (a potential hole or a localized region of deceased amplitude) is obtained:

$$
\rho=\frac{\sqrt{2 P / Q}}{L d} \sqrt{1-d^{2} \operatorname{sech}^{2}\left(\frac{\xi-U \tau}{L}\right)}
$$

where the parameter $d$ determines the depth of the modulation. For $d=1$ we get a dark soliton:

$$
\rho=\frac{\sqrt{2 P / Q}}{L d} \tanh \left(\frac{\xi-U \tau}{L}\right) .
$$

Thus the sign of the product $P Q$ determines the stability/instability profile of IAWs as well as the type of soliton structure. The soliton width is determined by the ratio $|P / Q|$.

We have numerically examined different parametric regions where some of the above excitations may occur. As the coefficients $P$ and $Q$ depend on nonthermal parameter $\beta$, ionto-electron temperature ratio $\sigma_{i}$, and positron-to-electron concentration ratio $\chi$, these parameters would definitely determine the modulational instability and the formation of envelope solitons. Numerical plots in Figures 1-3 show $P / Q$ as a function of $k$ for different values of $\beta, \sigma_{i}$, and $\chi$. It shows that the IAWs remain modulationally stable for $k$ less than certain critical value $k_{c}$ and for $k>k_{c}$ the wave is modulationally unstable.

In Figure 1 the variation of $P / Q$ with wave number has been plotted for different values of nonthermal parameter $(\beta)$, keeping positron concentration $(\chi)$ and ion temperature $\left(\sigma_{i}\right)$ fixed. It shows that as $\beta$ increases the value of critical wave number separating stable and unstable regions decreases. It is also noticed that as $\beta$ increases the width of the dark solitons increases, but that of the bright solitons decreases.

In Figure $2 P / Q$ is plotted as function of $k$ for different values of ion temperature $\left(\sigma_{i}\right)$ taking other plasma parameters such as positron concentration $(\chi)$ and nonthermal parame$\operatorname{ter}(\beta)$ as constant. It is seen that as $\sigma_{i}$ increases critical wave number decreases; the width of dark solitons increases but that of bright solitons decreases.

Figure 3 is a $P / Q$ versus wave number plot for different values of positron concentration $(\chi)$, keeping the values of nonthermal parameter $(\beta)$ and ion temperature $\left(\sigma_{i}\right)$ constant. It shows that as the value of $\chi$ increases the critical wave number increases. The width of dark solitons decreases and that of bright solitons increases as $\chi$ increases.

Qualitatively these results agree with those obtained by Gill et al. [31] but quantitatively there are differences. We find that the critical wave number is more sensitive to the variation in $\beta, \sigma_{i}$, and $\chi$ than that predicted by Gill et al. [31].

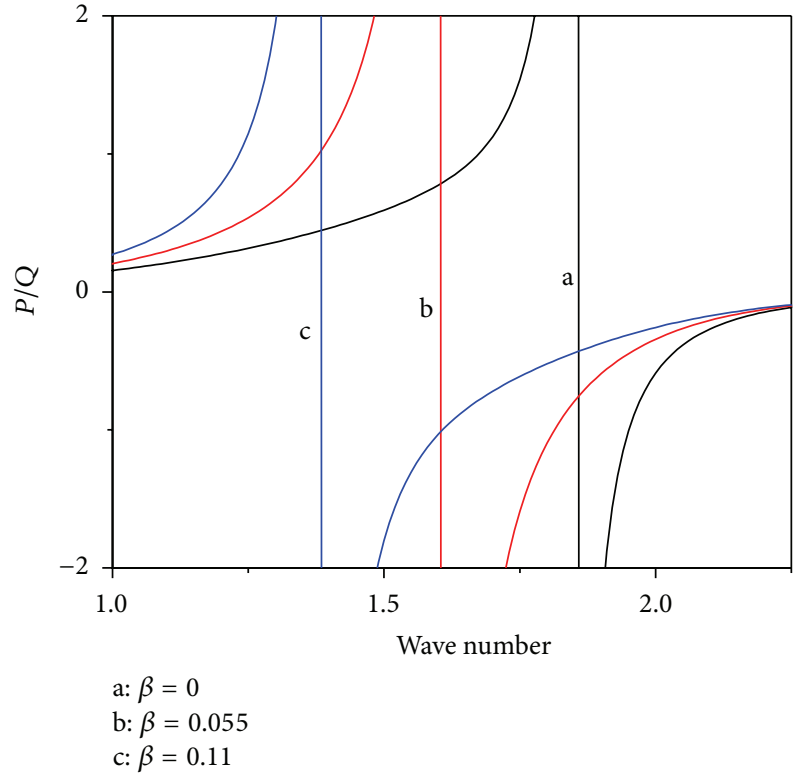

Figure 1: Plot of $P / Q$ versus wave number $k$ for different values of nonthermal parameter $(\beta)$. Curves labelled $a, b$, and $c$ correspond to $\beta=0,0.055$, and 0.11 , respectively. $\chi=0.22, \sigma_{p}=0.01$, and $\sigma_{i}=0.02$.

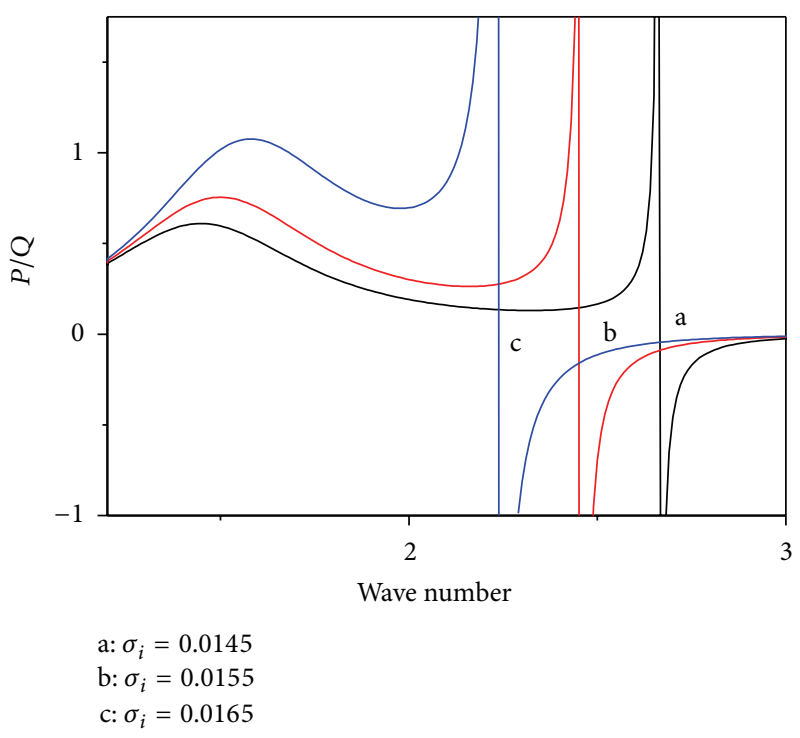

Figure 2: Plot of $P / Q$ versus wave number $k$ for different values of ion temperature $\left(\sigma_{i}\right)$. Curves labelled $\mathrm{a}, \mathrm{b}$, and $\mathrm{c}$ correspond to $\sigma_{i}=$ $0.0145,0.0155$, and 0.0165 , respectively. $\chi=0.2, \sigma_{p}=0.015$, and $\beta=0.022$.

In addition, we have numerically studied the dependence of growth rate of instability on all the plasma parameters $\beta, \sigma_{i}$, and $\chi$. The results are shown in Figures 4, 5, and 6. It is shown that the growth rate of instability increases with increase in the nonthermality of electrons and ion temperature but the increase of positron concentration reduces instability growth rate. 


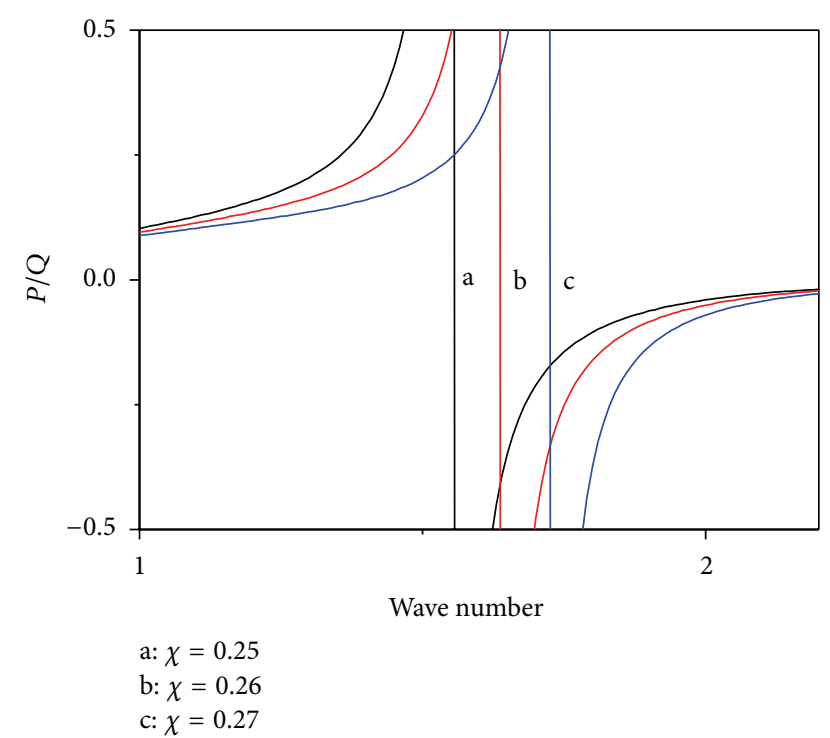

FIgURE 3: Plot of $P / Q$ versus wave number $k$ for different values of positron concentration $(\chi)$. Curves labelled $a, b$, and $c$ correspond to $\chi=0.25,0.26$, and 0.27 , respectively. $\beta=0.022, \sigma_{p}=0.01$, and $\sigma_{i}=0.052$.

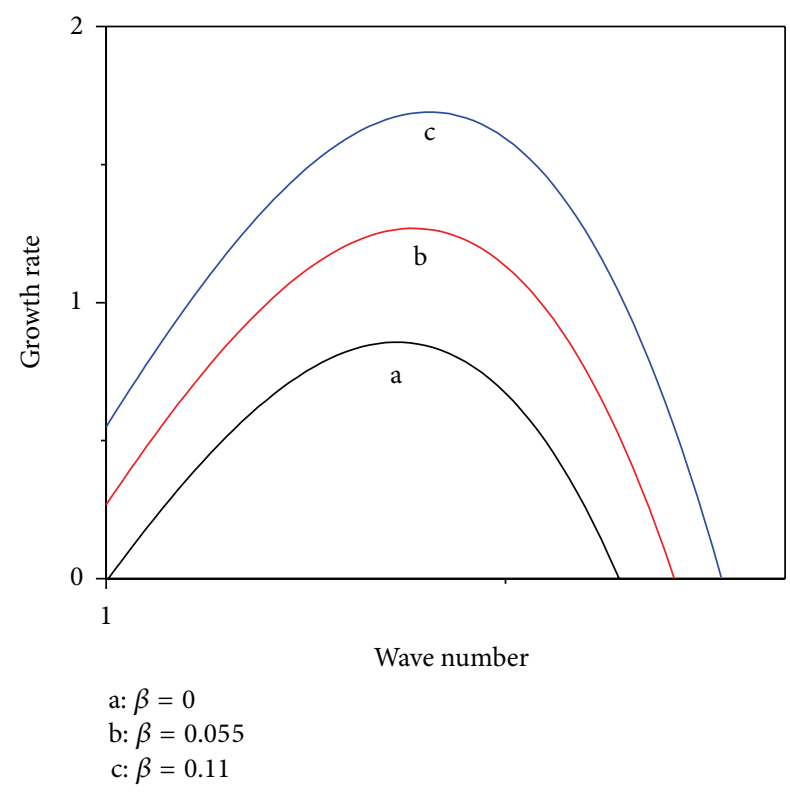

FIGURE 4: Plot of growth rate versus wave number $k$ for different values of nonthermal parameter $(\beta)$. Curves labelled $a, b$, and $c$ correspond to $\beta=0,0.055$, and 0.11 , respectively. $\chi=0.02, \sigma_{p}=$ 0.01 , and $\sigma_{i}=0.002$.

\section{Conclusions}

In the present work, we have investigated modulational instability and envelope excitations of IAWs in the $e$ - $p$ $i$ plasma in detail including simultaneously the effects of nonthermality of electrons and temperatures of ions. Our main findings are summarized below.

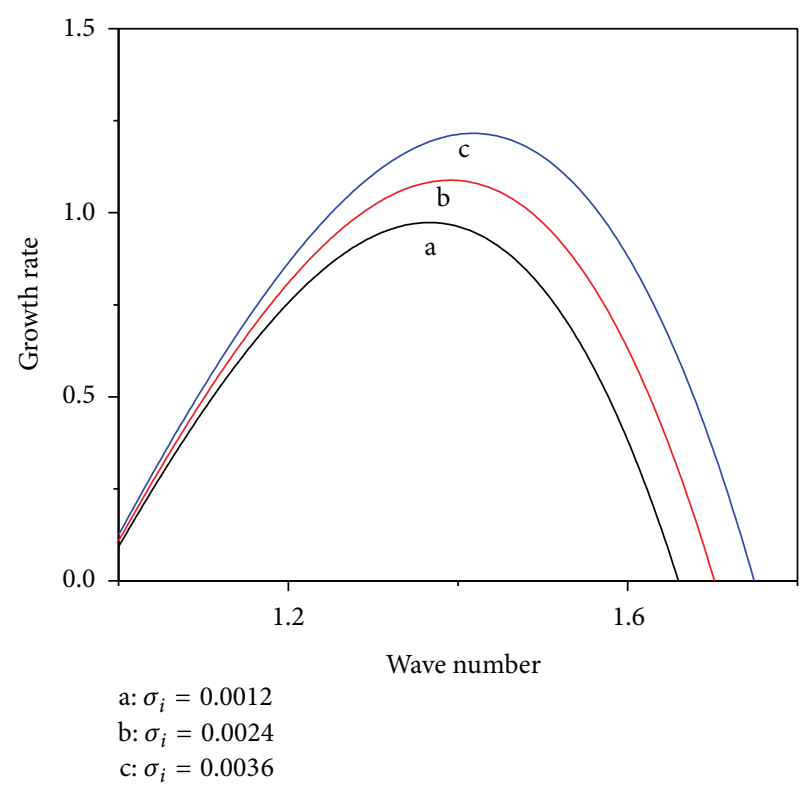

FIGURE 5: Plot of growth rate versus wave number $k$ for different values of ion temperature $\left(\sigma_{i}\right)$. Curves labelled $\mathrm{a}, \mathrm{b}$, and c correspond to $\sigma_{i}=0.0012,0.0024$, and 0.0036 , respectively. $\chi=0.001, \sigma_{p}=$ 0.01 , and $\beta=0.001$.

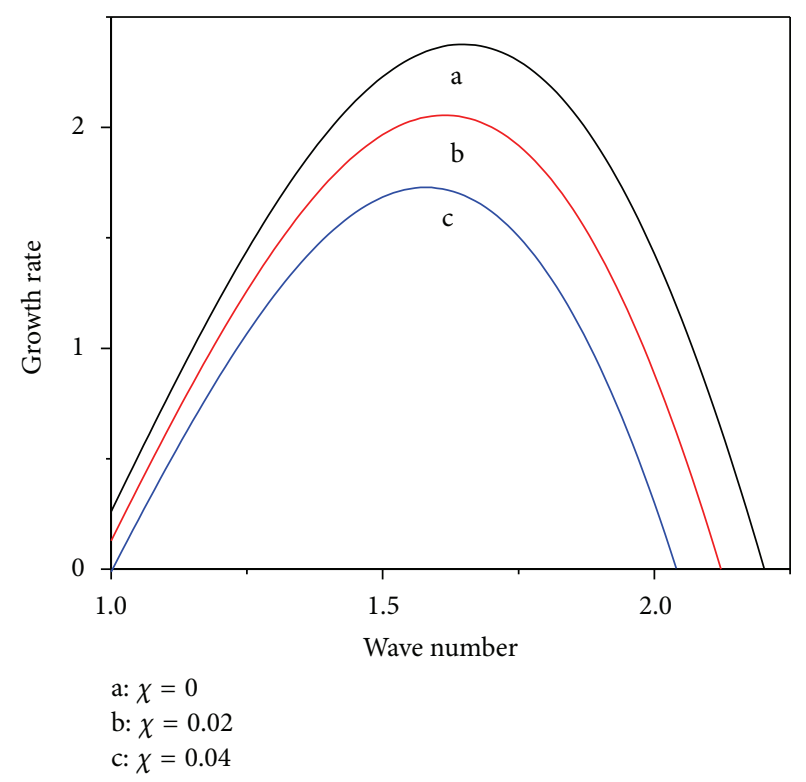

FIGURE 6: Plot of growth rate versus wave number $k$ for different values of positron concentration $(\chi)$. Curves labelled $a, b$, and $c$ correspond to $\chi=0,0.02$, and 0.04 , respectively. $\beta=0.01, \sigma_{p}=$ 0.01 , and $\sigma_{i}=0.01$.

(i) The wave frequency increases with increase in nonthermality of electrons and the temperature of ions whereas the increase in positron concentration decreases the wave frequency.

(ii) There exists a critical wave number $k_{c}$ below which the wave is modulationally stable and above which the wave is modulationally unstable. 
(iii) The value of the critical wave number and the characteristics of bright/dark envelope solitons depend significantly on the nonthermal parameter $(\beta)$, ion temperature $\left(\sigma_{i}\right)$, and positron concentration $(\chi)$.

Finally we would like to mention that the results presented in this paper may be useful to explain modulational instability and envelope soliton excitations of IAWs in some astrophysical and space environments where $e-p-i$ plasmas with nonthermal electrons are present.

\section{Conflict of Interests}

The authors declare that there is no conflict of interests regarding the publication of this paper.

\section{Acknowledgment}

The authors would like to thank the reviewers for various suggestions and helpful comments in bringing the paper to the present form.

\section{References}

[1] H. R. Miller and P. J. Witta, Active Galactic Nuclei, Springer, Berlin, Germany, 1978.

[2] F. C. Michel, "Theory of pulsar magnetospheres," Reviews of Modern Physics, vol. 54, no. 1, pp. 1-66, 1982.

[3] F. C. Michel, Theory of Neutron Star Magnetosphere, Chicago University Press, Chicago, Ill, USA, 1991.

[4] M. I. Barns, Positron Electron Pairs in Astrophysics, American Institute of Physics, New York, NY, USA, 1983.

[5] W. K. Misner, S. Thorne, and J. A . Wheeler, Gravitation, Freeman, San Francisco, Calif, USA, 1973.

[6] M. J. Rees, G. W. Gibbons, S. W. Hawking, and S. Siklaseds, The Early Universe, Cambridge University Press, Cambridge, UK, 1983.

[7] E. Tandberg-Hanssen and A. Gordon Emslie, The Physics of Solar Flares, Cambridge University Press, Cambridge, UK, 1988.

[8] A. Cairns, R. Bingham, R. O. Dendy, C. M. C. Nairn, P. K. Shukla, and A. A. Mamun, "Ion sound solitary waves with density depressions," Journal of Physics IV. France, vol. 5, no. C6, pp. 43-48, 1995.

[9] S. I. Popel, S. V. Vladimirov, and P. K. Shukla, "Ion-acoustic solitons in electron-positron-ion plasmas," Physics of Plasmas, vol. 2, no. 3, pp. 716-719, 1995.

[10] Y. N. Nejoh, "The effect of the ion temperature on large amplitude ion-acoustic waves in an electron-positron-ion plasma," Physics of Plasmas, vol. 3, no. 4, pp. 1447-1451, 1996.

[11] H. R. Pakzad, "Ion acoustic solitary waves in plasma with nonthermal electron, positron and warm ion," Astrophysics and Space Science, vol. 323, no. 4, pp. 345-350, 2009.

[12] S. Ghosh and R. Bharuthram, "Ion acoustic solitons and double layers in electron-positron-ion plasmas with dust particulates," Astrophysics and Space Science, vol. 314, no. 1-3, pp. 121-127, 2008.

[13] N. Jehan, W. Masood, and A. M. Mirza, "Planar and nonplanar dust acoustic solitary waves in electronpositron-ion- dust plasmas," Physica Scripta, vol. 80, no. 3, Article ID 035506, 2009.

[14] R. A. Cairns, A. A. Mamun, R. Bingham, and P. K. Shukla, "Ion acoustic solitons in a magnetised plasma with nonthermal electrons," Physica Scripta, vol. 63, pp. 80-86, 1996.
[15] B. Ghosh, S. Banerjee, and S. N. Paul, "Effect of non-thermal electrons and warm negative ions on ion-acoustic solitary waves in multi-component drifting plasma," Indian Journal of Pure and Applied Physics, vol. 51, no. 7, pp. 488-493, 2013.

[16] B. Ghosh, S. N. Paul, C. Das, I. Paul, and S. Banerjee, "Electrostatic double layers in a multicomponent drifting plasma having nonthermal electrons," Brazilian Journal of Physics, vol. 43, no. 1-2, pp. 28-33, 2013.

[17] P. O. Dovner, A. I. Eriksson, R. Bostrom, and B. Holback, "Freja multiprobe observations of electrostatic solitary structures," Geophysical Research Letters, vol. 21, no. 17, pp. 1827-1830, 1994.

[18] R. Boström, G. Gustafsson, B. Holback, G. Holmgren, H. Koskinen, and P. Kintner, "Characteristics of solitary waves and weak double layers in the magnetospheric plasma," Physical Review Letters, vol. 61, no. 1, pp. 82-85, 1988.

[19] R. A. Cairns, A. A. Mamun, R. Bingham et al., "Electrostatic solitary structures in non-thermal plasmas," Geophysical Research Letters, vol. 22, no. 20, pp. 2709-2712, 1995.

[20] M. Salahuddin, H. Saleem, and M. Saddiq, "Ion-acoustic envelope solitons in electron-positron-ion plasmas," Physical Review E, vol. 66, no. 3, Article ID 036407, 2002.

[21] T. S. Gill, C. Bedi, and A. S. Bains, "Envelope excitations of ion acoustic solitary waves in a plasma with superthermal electrons and positrons," Physica Scripta, vol. 81, no. 5, Article ID 055503, 2010.

[22] G. Murtaza and M. Salahuddin, "Modulational instability of ion acoustic waves in a magnetised plasma," Plasma Physics, vol. 24, no. 5, pp. 451-456, 1982.

[23] Yashvir, T. N. Bhatnagar, and S. R. Sharma, "Nonlinear ionacoustic waves and solitons in warm-ion magnetized plasma," Plasma Physics and Controlled Fusion, vol. 26, no. 11, article 004, pp. 1303-1310, 1984.

[24] J. K. Chawla, M. K. Mishra, and R. S. Tiwari, "Modulational instability of ion-acoustic waves in electron-positron-ion plasmas," Astrophysics and Space Science, vol. 347, pp. 283-292, 2013.

[25] T. K. Baluku and M. A. Hellberg, "Ion acoustic solitary waves in an electron-positron-ion plasma with non-thermal electrons," Plasma Physics and Controlled Fusion, vol. 53, no. 9, Article ID 095007, 2011.

[26] A. E. Dubinov and M. A. Sazonkin, "Nonlinear theory of ionacoustic waves in an electron-positron-ion plasma," Plasma Physics Reports, vol. 35, no. 1, pp. 14-24, 2009.

[27] S. Mahmood, S. Siddiqui, and N. Jehan, "Modulational instability of ion acoustic wave with warm ions in electron-positron-ion plasmas," Physics of Plasmas, vol. 18, no. 5, Article ID 052309, 2011.

[28] A. S. Bains, N. S. Saini, and T. S. Gill, "Modulational instability of ion-acoustic soliton in electron-positron-ion plasma with dust particulates," Astrophysics and Space Science, vol. 343, no. 1, pp. 293-299, 2013.

[29] P. Eslami, M. Mottaghizadeh, and H. R. Pakzad, "Modulational instability of ion acoustic waves in e-p-i plasmas with electrons and positrons following a q-nonextensive distribution," Physics of Plasmas, vol. 18, no. 10, Article ID 102313, 2011.

[30] J. Zhang, Y. Wang, and L. Wu, "Modulation instability of ion acoustic waves, solitons, and their interactions in nonthermal electron-positron-ion plasmas," Physics of Plasmas, vol. 16, no. 6, Article ID 062102, 2009.

[31] T. S. Gill, A. S. Bains, N. S. Saini, and C. Bedi, "Ion-acoustic envelope excitations in electron-positron-ion plasma with nonthermal electrons," Physics Letters A, vol. 374, no. 31-32, pp. $3210-3215,2010$. 
[32] B. Ghosh, S. N. Paul, C. Das, and I. Paul, "Modulational instability of high frequency surface waves on warm plasma half-space," Canadian Journal of Physics, vol. 90, no. 3, pp. 291297, 2012.

[33] B. Ghosh and K. P. Das, "Modulational instability of electron plasma waves in a cylindrical wave guide," Plasma Physics and Controlled Fusion, vol. 27, no. 9, pp. 969-982, 1985.

[34] B. Ghosh, S. Chandra, and S. N. Paul, "Amplitude modulation of electron plasma waves in a quantum plasma," Physics of Plasmas, vol. 18, no. 1, Article ID 012106, 2011.

[35] H. R. Pakzad, "Ion acoustic solitary waves in plasma with nonthermal electron and positron," Physics Letters A: General, Atomic and Solid State Physics, vol. 373, no. 8-9, pp. 847-850, 2009.

[36] T. Kakutani and N. Sugimoto, "Krylov-Bogoliubov-Mitropolsky method for nonlinear wave modulation," The Physics of Fluids, vol. 17, pp. 1617-1625, 1974. 

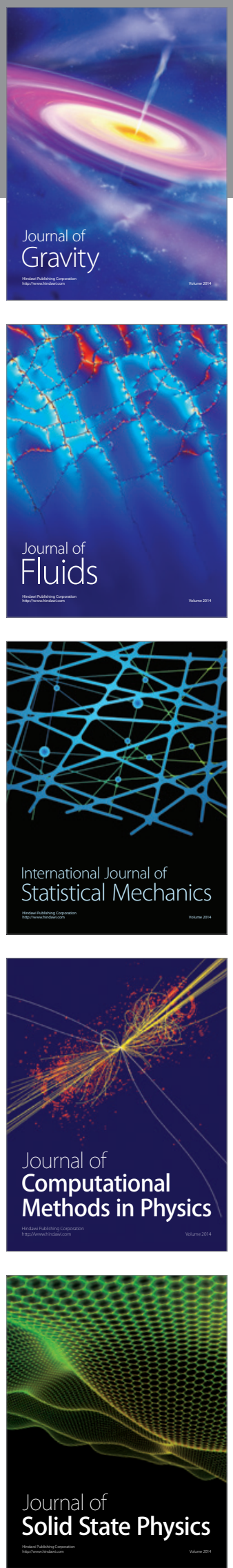

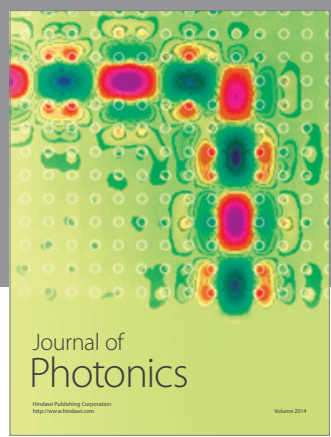

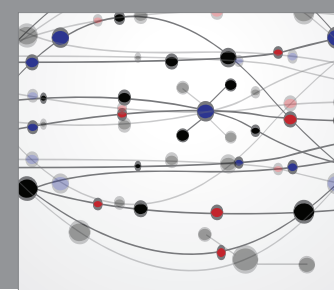

The Scientific World Journal

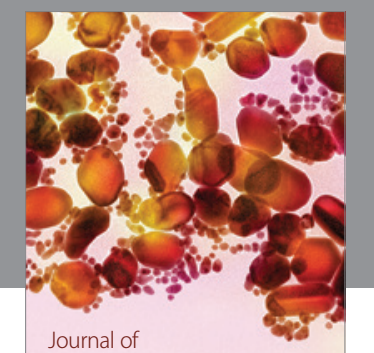

Soft Matter
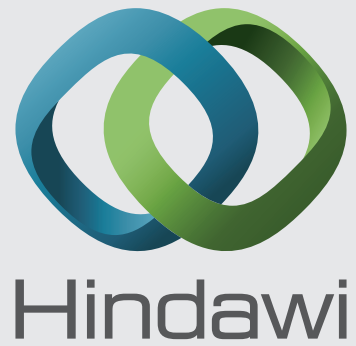

Submit your manuscripts at

http://www.hindawi.com
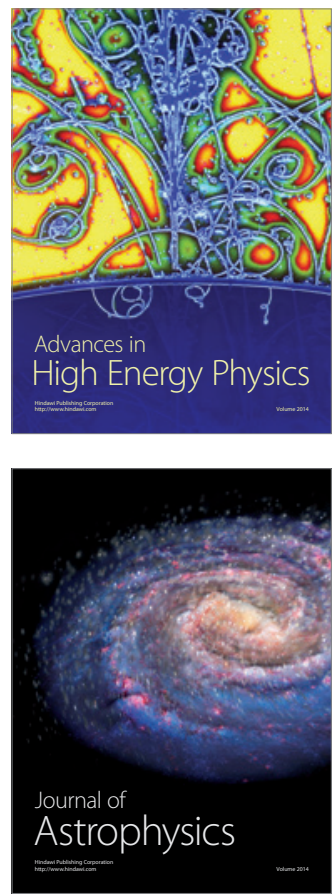
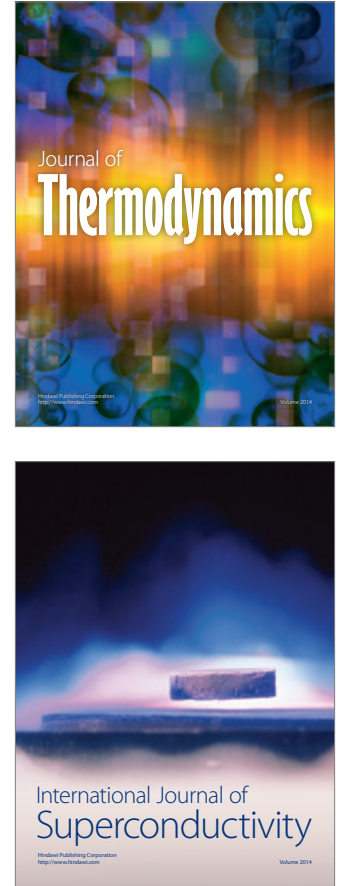
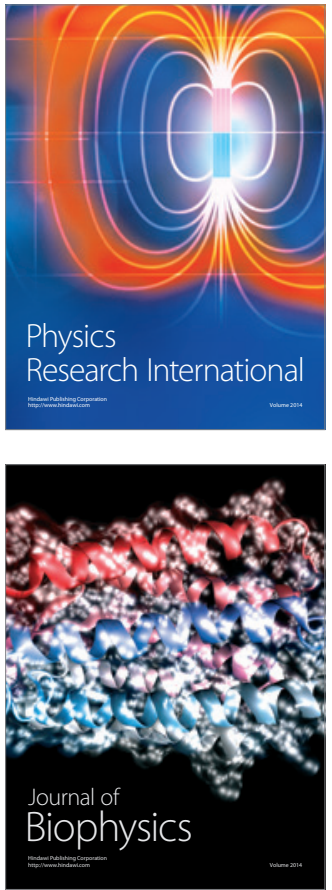
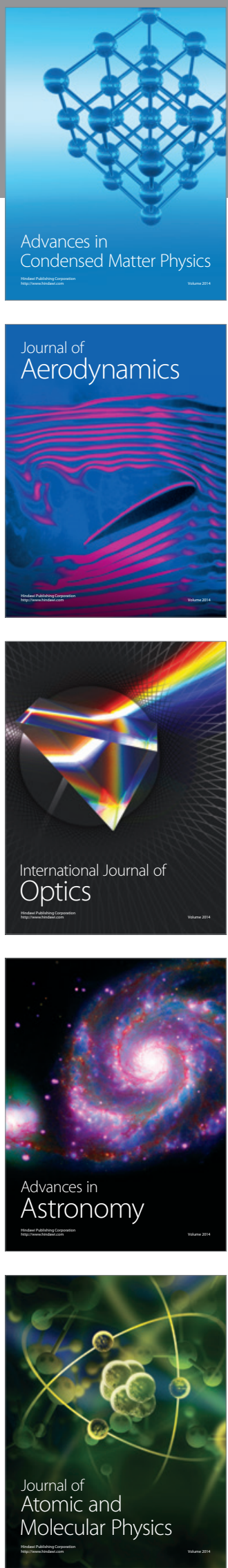